\title{
Simplified modeling of sprinkler head fluid mechanics
}

\author{
T. M. Myers, A. W. Marshall \& H. R. Baum \\ University of Maryland, College Park, USA
}

\begin{abstract}
Understanding the atomization of fire sprinkler sprays fills a critical gap in the modeling of fire suppression systems. Previous research by the authors has shown instability models and spatio-stochastic transport models can paint most of the sprinkler spray picture, but require input in the form of thickness and velocity of unstable fluid sheets. The model outlined makes use of a free streamline model of the flow which can be constructed based on a description of the water jet as a potential flow. The free stream-lines separating the jet from the surrounding air take the form of vortex sheets with the air assumed to be at rest. Any solution so obtained is an exact solution of inviscid flow equations with interior flow an exact solution to the Navier-Stokes equations. There is considerable literature describing two-dimensional free streamline flows, with most problems being solved through the use of the hodograph method. In three dimensions, the hodograph method and complex variable techniques are no longer available.Instead a mathematical model can be formulated using Green's functions in conjunction with free streamline constant pressure assumptions regarding the surface of the flow. The resulting mathematical model allows for the determination of a flow field over a sprinkler head of arbitrary geometry and input conditions. Knowledge of the sprinkler head flow field provides insight into the impact of sprinkler head geometry and fluid velocity as well as providing the necessary inputs for a complete spatio-stochastic model of fire sprinkler sprays.

Keywords: atomization, fire, fluid potential, free stream, Green's function, spray, sprinkler.
\end{abstract}




\section{Introduction}

Sprinkler systems are a ubiquitous form of fire protection. Despite their widespread use, analytical models to predict their performance have yet to be developed. Furthermore, few detailed measurements to support the development of these models are available. The performance of sprinkler sprays depends on the spray generated by the sprinkler, the dispersion of the spray within the flames, and the wetting of burning surfaces. Each one of these stages involves complex transport processes resulting in important modeling and measurement challenges. The current study focuses on developing models to predict the spray generated by the sprinkler. Sprinkler sprays are generated in an impinging jet configuration as shown in figure 1 . The jet is transformed into a thin sheet as it impinges on the deflector. This thin sheet becomes unstable as it moves radially outward away from the deflector and ultimately breaks up into ring-like ligaments which disintegrate into drops. The breakup of the sheet into drops is largely determined by the thickness and velocity of the sheet at the deflector edge. In fact, physics based stability models are available for predicting the sheet breakup from these parameters (Wu et al. [1]). As a first step in predicting spray generation, a free surface flow model is explored in the current study to predict the transformation of the jet into thin sheets as it interacts with the deflector. This free surface model will provide the important sheet thickness and velocity information required to predict sheet breakup and ultimately drop formation.

The essential notion is to make use of the fact that a free streamline model of the flow can be constructed based on the description of the water jet as a velocity potential. Using the potential flow assumptions, the fluid velocity potential solutions can be reduced to a boundary value problem. By forcing the velocity potential to satisfy Laplace's equation and specifying the values on all boundaries, a complete velocity potential field within the boundaries may be realized. Any solution so obtained is an exact solution of the inviscid flow equations, and the interior flow is an exact solution of the Navier-Stokes equations.

The free streamlines separating the water jet from the surrounding air are taken to be vortex sheets, and the air is assumed to be at rest. The method relies on the fact that a Green's function satisfying the potential flow equation and satisfying appropriate boundary and symmetry conditions can be found. In mathematics, a Green's function is a specific type of function used to solve inhomogeneous differential equations. It is a function which transforms a boundary value of a function into the function's response to the boundary value in all space.

There is a considerable literature describing two-dimensional free streamline flows dating back to the late nineteenth century. These problems have typically been addressed using the hodograph method, which uses the velocity components as independent variables. Several of the classical solutions are described by Lamb [2] and Batchelor [3]. These flows are typically characterized by the fact that the solid boundaries are composed of straight line segments, while the free surface is a streamline at a constant pressure. These qualities make i-t relatively easy to determine the boundary shape in the hodograph plane, and the fact that 


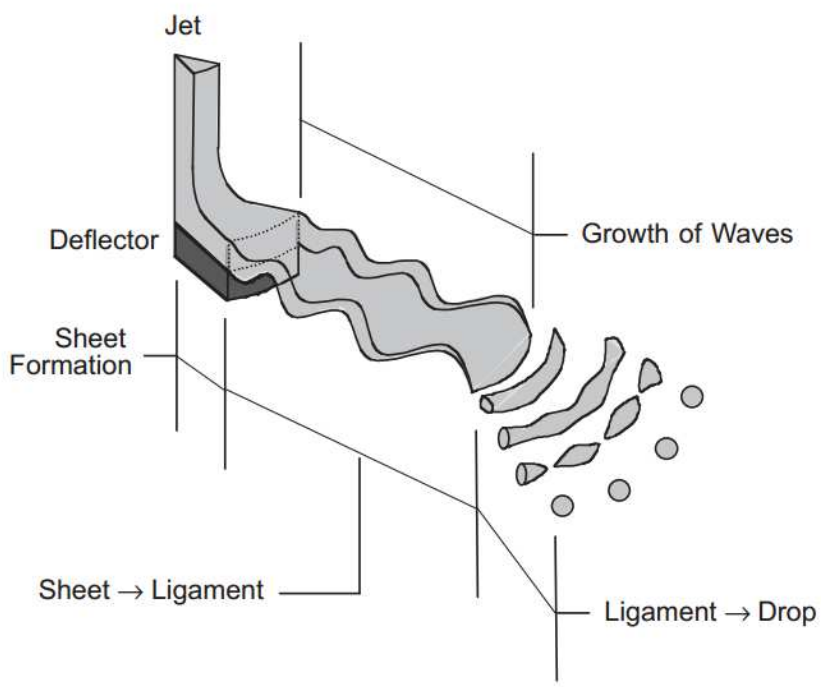

Figure 1: Description of atomization process (sheet formation, sheet trajectory, sheet break-up, ligament break-up) (Wu et al. [1]).

the velocity components are functions of a complex variable makes conformal mapping a powerful tool in constructing the solution.

In three dimensions the hodograph method is no longer available, nor is the use of complex variable techniques. However, the potential flow still satisfies the Laplace equation, and the free streamline is still a constant pressure surface. Thus, formulation of the boundary value problem in terms of a Green's function reduces the problem to the determination of the shape of the free surface and the outflow conditions on the deflector plate. This latter problem can be greatly simplified if the two-dimensional solution for the flow through a bounded orifice (see Steckler et al. [4]) is used to represent the outflow through the slot in each segment. The mathematical formulation of this approximate model is discussed next.

\section{Mathematical model}

To begin, total flow through the boundaries of a sprinkler can be described by:

$$
\begin{gathered}
\dot{Q}_{\text {total }}=\dot{Q}_{\text {tine }}+\dot{Q}_{\text {slot }} \\
\dot{Q}_{\text {total }}=\alpha \dot{Q}_{\text {slot }}
\end{gathered}
$$

where $\dot{Q}_{\text {total }}$ is the total flow impinging upon the sprinkler in the form of a jet, $\dot{Q}_{\text {tine }}$ is the total flow deflected by the sprinkler deflector plate, $\dot{Q}_{\text {slot }}$ is the flow 
which passes through perforations in the deflector plate and $\alpha$ is the flow split, which defines the fraction of the flow which passes through the deflector plate.

All flow composing $\dot{Q}_{\text {total }}$ enters in the form of a jet of radius, $R_{j}$, and with a uniform downward velocity, $U_{j}$. The entire problem can be posed in a cylindrical coordinate system as follows:

$$
\vec{r}=(x, y, z)=(r \cos (\theta), r \sin (\theta), z)
$$

The system in eqn (3) can then be nondimensionalized with $R_{j}$, resulting in:

$$
\overrightarrow{r^{*}}=(x, y, z)=\left(\frac{r}{R_{j}} \cos (\theta), \frac{r}{R_{j}} \sin (\theta), \frac{z}{R_{j}}\right)
$$

Further, velocity field and potential can be nondimensionalized as follows:

$$
\overrightarrow{u^{*}}\left(\overrightarrow{r^{*}}\right)=\frac{\vec{u}\left(\overrightarrow{r^{*}}\right)}{U_{j}} \quad \phi^{*}\left(\overrightarrow{r^{*}}\right)=\frac{\phi\left(\overrightarrow{r^{*}}\right)}{R_{j} U_{j}}
$$

For the remainder of the paper, we will drop the star notation of nondimensionalization.

The starting point is the assumption that the impinging jet velocity field $\vec{u}(\vec{r})$ can be described as a potential flow, satisfying the equation:

$$
\nabla^{2} \phi(\vec{r})=0 \quad \vec{u}(\vec{r})=\nabla \phi(\vec{r})
$$

The local pressure change $p-p_{\infty}$ is determined by the Bernoulli equation:

$$
\rho u^{2} / 2+p-p_{\infty}=H=\rho U_{I}^{2} / 2
$$

With these preliminaries established, the sprinkler boundary value problem can now be formulated. The Green's function we seek must be a solution to the following equation:

$$
\nabla^{2} G\left(\vec{r}, \vec{r}_{o}\right)=\delta\left(\vec{r}-\vec{r}_{o}\right)
$$

Here $\overrightarrow{r_{o}}$ denotes the location of the mathematical source required by the Green's function and $\delta$ denotes the Dirac Delta function in three space dimensions. Now consider the integral $I(\vec{r})$ defined by:

$$
I(\vec{r})=\int\left[G\left(\vec{r}, \vec{r}_{o}\right) \nabla^{2} \phi\left(\vec{r}_{o}\right)-\phi\left(\vec{r}_{o}\right) \nabla^{2} G\left(\vec{r}, \vec{r}_{o}\right)\right] d^{3} \vec{r}_{o}
$$

The integral is taken over the entire volume encompassing the domain on which the problem is posed. In the present case the volume is bounded by the surface of the deflector plate and the free surface of the impinging water jet. The integral $I(\vec{r})$ will now be evaluated in two different ways. First, note that from eqns (6) 
and (8):

$$
I(\vec{r})=-\phi(\vec{r})
$$

Second, $I(\vec{r})$ can be written in the form:

$$
I(\vec{r})=\int \nabla \cdot\left[G\left(\vec{r}, \vec{r}_{o}\right) \nabla \phi\left(\vec{r}_{o}\right)-\phi\left(\vec{r}_{o}\right) \nabla G\left(\vec{r}, \vec{r}_{o}\right)\right] d^{3} \vec{r}_{o}
$$

Then, using the Divergence theorem:

$$
I(\vec{r})=\oint\left[G\left(\vec{r}, \vec{r}_{s}\right) \frac{\partial \phi}{\partial n}\left(\vec{r}_{s}\right)-\phi\left(\vec{r}_{s}\right) \frac{\partial G}{\partial n}\left(\vec{r}, \vec{r}_{s}\right)\right] d^{2} s
$$

The integral in eqn (12) is taken over the surface that bounds the volume of interest. Here, $n$ is a local coordinate normal to the bounding surface pointing outward from the volume, and $\vec{r}_{s}$ denotes a point on that surface. finally, eliminating $I(\vec{r})$ from the two expressions, a relation between $\phi(\vec{r})$, its value on the boundary, the fluid velocity normal to the boundary, and the Greens function, is obtained.

$$
\phi(\vec{r})=\oint\left[\phi\left(\vec{r}_{s}\right) \frac{\partial G}{\partial n}\left(\vec{r}, \vec{r}_{s}\right)-G\left(\vec{r}, \vec{r}_{s}\right) \frac{\partial \phi}{\partial n}\left(\vec{r}_{s}\right)\right] d^{2} s
$$

The result presented above is very general, as no assumptions regarding the specific boundary conditions or the shape of the boundary are needed to obtain it. To proceed further, it is necessary to specify the information available to formulate a specific boundary value problem relevant to the sprinkler jet impingement on a given deflector plate. This requires a specific choice of Green's function. The starting point is the observation that the simplest Greens function satisfying eqn (8), denoted here by $G_{0}\left(\vec{r}, r_{o}, \theta_{o}, z_{o}\right)$, is:

$$
G_{0}\left(\vec{r}, r_{o}, \theta_{o}, z_{o}\right)=-\frac{1}{4 \pi} \frac{1}{\left|\vec{r}-\vec{r}_{o}\right|}
$$

This solution does not satisfy any boundary conditions on any surface a finite distance from the source. Next consider a source located a distance $z_{o}$ above a planar barrier at $z=0$ with prescribed outflow velocity through specified segments of the barrier. The appropriate Green's function is then:

$$
G_{1}\left(\vec{r}, r_{o}, \theta_{o}, z_{o}\right)=G_{0}\left(\vec{r}, r_{o}, \theta_{o}, z_{o}\right)+G_{0}\left(\vec{r}, r_{o}, \theta_{o},-z_{o}\right)
$$

The new solution certainly satisfies eqn (8) in the half space above $z=0$ and also satisfies the condition of no normal gradient at the surface $z_{o}=0$. Thus, since the velocity component normal to the barrier either vanishes or is prescribed everywhere, the first term in eqn (11) vanishes and the solution everywhere in $z>0$ is given by the integral over those portions of the boundary where the outflow velocity is known, with $G$ replaced by $G_{1}$. Note that $G_{1}\left(\vec{r}, r_{o}, \theta_{o}, 0\right)=$ $2 G_{0}\left(\vec{r}, r_{o}, \theta_{o}, 0\right)$. 
Based upon symmetry of the specific problem, the Green's function given above can be further adapted to capture appropriate boundary conditions. The above is a general mathematical model suitable for all perforated deflector problems. In the following section we will assume an axisymmetric simplification in order to explore the possible forms of various boundary conditions.

\section{Axisymmetric simplification}

The above mathematical model is complete, but quite general. By making a few assumptions, including that the sprinkler is axisymmetric and that slot flow can be represented as two dimensional, we are able to explore boundary conditions and their implications for the more general problem. Many of the geometric effects induced by the details of the deflector plate geometry are lost in this approach, but the influence of three basic dimensionless parameters can still be accounted for. They are: the ratio of the impinging jet radius, $R_{j}$, to that of the deflector plate, $R_{p}$, the ratio of the area of the openings in the deflector plate, $A_{o}$, to the nominal area of the deflector plate, $\pi R_{p}^{2}$, and the ratio of the radial position of the centroid of the plate openings, $R_{c}$, to the deflector plate radius. The discrete openings in the actual plate are smeared out uniformly with respect to the angular coordinate $\theta$ and the radial location and width of the smeared locations can be chosen to match the last two parameters mentioned above.

Once this task has been performed, the boundary conditions of $\phi\left(\vec{r}_{s}\right)$ and its normal gradient $\vec{n} \cdot \nabla \phi\left(\vec{r}_{s}\right)$ appearing in eqn (13) are independent of $\theta$. All of the bounding surfaces are now figures of revolution, and the only quantities containing an angular dependence are the Green's function and its normal derivative.

The appropriate Green's function is now given by the angular integration of the Green's function presented in the previous section.

$$
\mathcal{G}\left(\vec{r}, r_{o}, z_{o}\right)=-\frac{1}{4 \pi} \int_{0}^{2 \pi} \frac{1}{\sqrt{r^{2}+r_{o}^{2}-2 r r_{o} \cos \left(\theta-\theta_{o}\right)+\left(z-z_{o}\right)^{2}}} d \theta_{o}
$$

The quantity $\mathcal{G}$ physically represents a ring source of fluid situated at $r=r_{o}$, $z=z_{o}$. Similarly, $\mathcal{G}_{1}$ represents a ring source situated at $r=r_{o}, z=z_{o}$ located in a semi-infinite space bounded below by the plane $z=0$, where $\mathcal{G}_{1}$ was obtained in the same manner from $\mathcal{G}$ that $G_{1}$ was obtained from $G$ in the previous section. These functions can be evaluated in terms of complete elliptic integrals of the first and second kinds, denoted respectively as $K(m)$ and $E(m)$.

$\mathcal{G}$ can be written explicitly in the form:

$$
\begin{gathered}
\mathcal{G}\left(\vec{r}, r_{o}, z_{o}\right)=-\frac{1}{\pi \sqrt{\left(r+r_{o}\right)^{2}+\left(z-z_{o}\right)^{2}}} K(m) \\
m=\frac{4 r r_{o}}{\left(r+r_{o}\right)^{2}+\left(z-z_{o}\right)^{2}}
\end{gathered}
$$


Figures 2 and 3 show the contour plot and streamlines associated with $\mathcal{G}_{1}$ for a particular ring source.

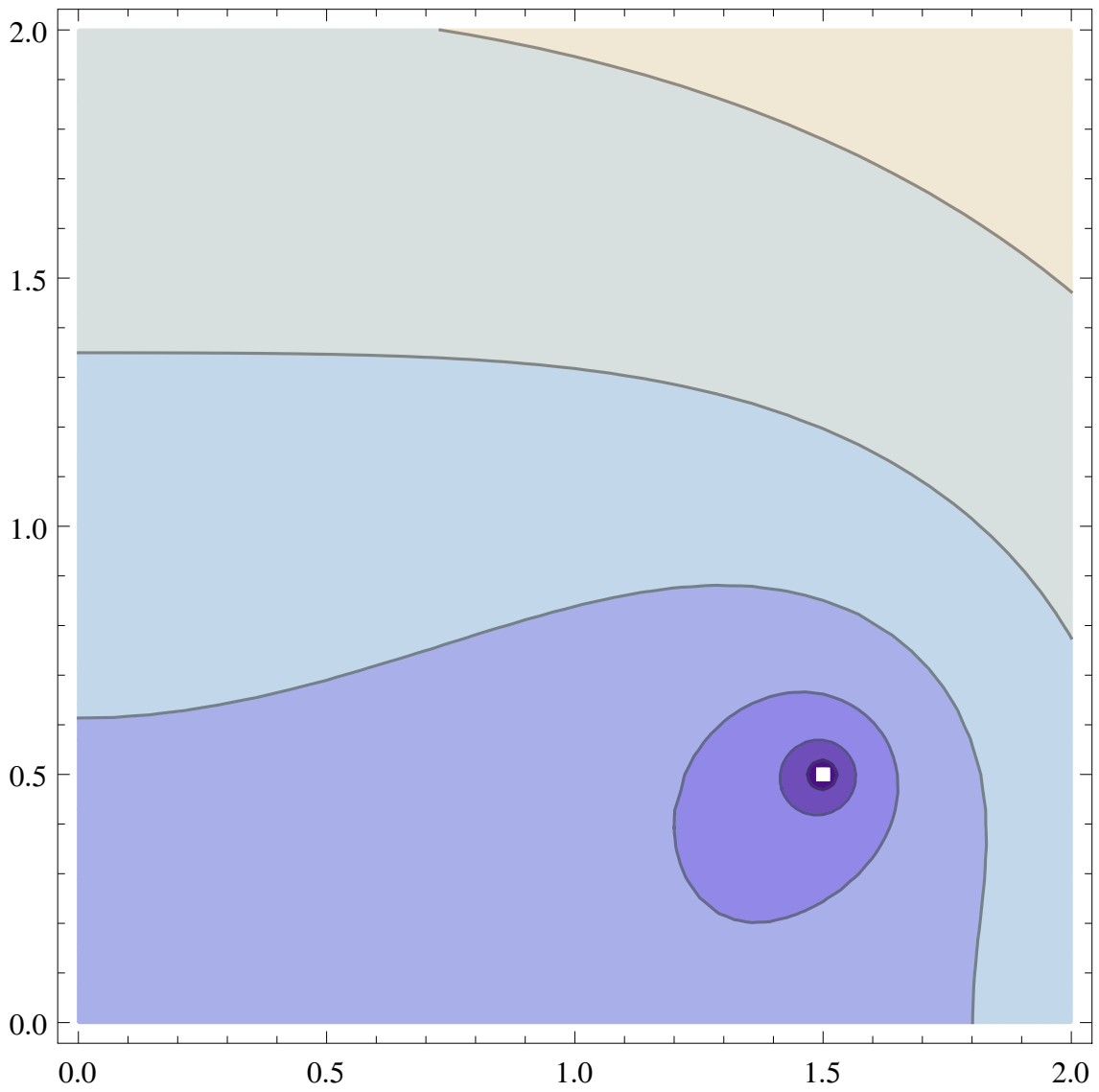

Figure 2: Plot of the Greens function $\mathcal{G}_{1}$ for a ring source placed at $r_{o}=1.5 ; z_{o}=$ 0.5 .

\subsection{Boundary values}

The bounding surface consists of four parts; an inlet disk of radius $R_{j}$, the bounding free streamline surface, a vertical cylinder of radius $R_{p}$ and height $h$ (the vertical distance between the bounding free streamline and the deflector plate) where the flow that does not pass through the deflector plate exits, and the deflector plate itself. Because of the formulation of the problem as a surface integral, each individual boundary can be evaluated individually, and summed to construct the 


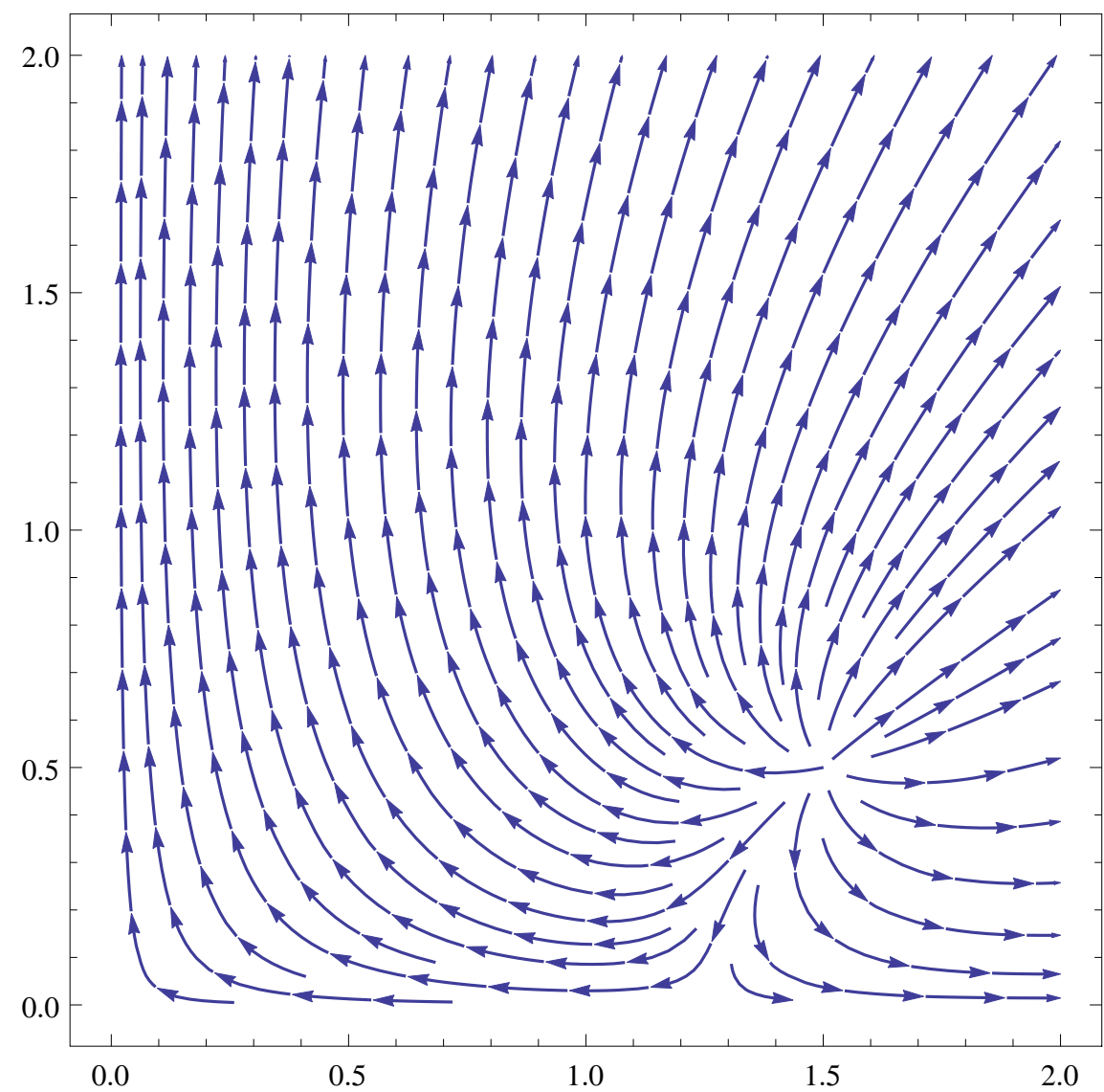

Figure 3: Streamlines for the velocity field associated with of the Greens function $\mathcal{G}_{1}$ for a ring source placed at $r_{o}=1.5 ; z_{o}=0.5$.

entire integral as follows:

$$
\phi(\vec{r})=-\left(I_{j}(\vec{r})+I_{t}(\vec{r})+I_{f s}(\vec{r})+I_{p}(\vec{r})\right)
$$

where $I_{j}(\vec{r}), I_{t}(\vec{r}), I_{f s}(\vec{r})$, and $I_{p}(\vec{r})$ correspond to the integral $I(\vec{r})$, as defined in eqn (12), evaluated on the surface of the inlet jet, tine (or flow that does not exit through the deflector plate), free stream, and flow through the plate, respectively.

The first among these, $I_{j}(\vec{r})$, is evaluated at the inlet. An approximation of the inlet flow as an axisymmetric jet with uniform velocity is appropriate for the sprinkler problem. The inlet boundary can be taken to span $0<r<1$ at a constant height $z=L_{j} / R_{j}$. Following from eqn (6) we see that $\phi$ can be set to 0 at one arbitrary location without having an impact on the physical results of the calculations. The inlet, having a uniform potential across the evaluated boundary, 
is an appropriate location. $\partial \phi / \partial n$ has a value of -1 , following from the definition of the chosen non dimensionalization. $I_{j}(\vec{r})$ can be written as follows:

$$
I_{j}(\vec{r})=\int_{0}^{1}-\mathcal{G}_{1}\left(r, L_{j} / R_{j}\right) d r
$$

Similarly, the flow exiting from the deflector plate, or $\dot{Q}_{\text {tine }}$, can be approximated as a sheet of uniform velocity and fixed height above the deflector plate, $h$. The tine boundary, $I_{t}(\vec{r})$, then exists at $r=R_{p} / R_{j}$ and spans $0<z<h$. $\partial \phi / \partial n$ has a uniform value of 1 , following from the free stream approximation, and $\phi$ is thus equal to $s\left(R_{p} / R_{j}\right)$, where $s(r)$ is the arc length of the free stream from $r=1$ to the radius, $r$. $I_{t}(\vec{r})$ can be written as follows:

$$
I_{t}(\vec{r})=\int_{0}^{h / R_{j}} \mathcal{G}_{1}\left(R_{p} / R_{j}, z\right)-s\left(R_{p} / R_{j}\right) \frac{\partial \mathcal{G}_{1}}{\partial n}\left(R_{p} / R_{j}, z\right) d z
$$

The surface integral over the free surface, $I_{f s}(\vec{r})$, spans from the nondimensional position $\left(r=1, z=L_{j} / R_{j}\right)$ to $\left(r=R_{p} / R_{j}, z=h / R_{j}\right)$. A prediction for the shape of the free surface can be reached through a simple analysis of the problem. From a simple mass balance we see that the stream line should have the form:

$$
r z=\frac{1-\alpha}{2}
$$

Performing an asymptotic analysis for $z>>1$ :

$$
r-1=A_{1} J_{1}\left(\lambda_{1}\right) e^{-\lambda_{1} z}
$$

where $A_{1}$ is some constant, $J_{1}$ is the Bessel function of the first kind and $\lambda_{1}$ is the first root of $J_{0}$ such that $J_{0}\left(\lambda_{1}\right)=0$.

From analysis valid where $r>>1$ and as $z \rightarrow 0$ the flow field can be approximated by an expansion of the Laplacian.

$$
\begin{gathered}
\frac{\partial^{2} \phi}{\partial z^{2}}+\frac{1}{r} \frac{\partial}{\partial r}\left(r \frac{\partial \phi}{\partial r}\right)=0 \\
\phi=r+\frac{f_{1}(z)}{r}+\frac{f_{2}(z)}{r^{3}}+\cdots
\end{gathered}
$$

The flow field can then be approximated:

$$
\phi=r-\frac{1}{2} \frac{z}{r}+\frac{1}{4 !} \frac{z^{4}}{r^{3}}+\mathcal{O}\left(\frac{1}{r^{5}}\right)
$$

If we postulate a smooth shape with asymptotic results we can guess the free surface has the form:

$$
r-1=A\left(\frac{1-\alpha}{2}\right) \frac{1}{\sinh \left(\lambda_{1} z\right)}
$$

where $A$ is a constant ensuring that eqn (27) conserves mass in the large $z$ limit. Figure has a plot showing the predicted free surface for $\alpha=0.5$ and an inlet height and deflector radius of ratios $L_{j} / R_{j}=R_{p} / L_{j}=3$. 


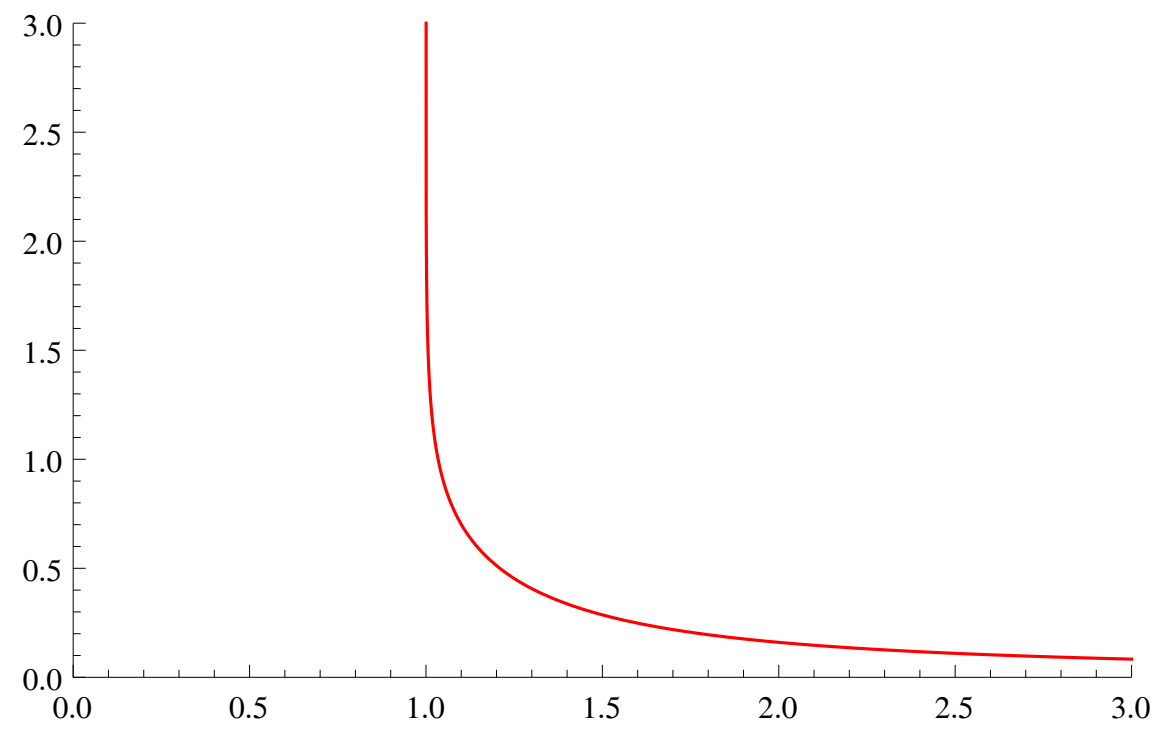

Figure 4: Shape of the free surface for $\alpha=0.5$ and an inlet height and deflector radius of ratios $L_{j} / R_{j}=R_{p} / L_{j}=3$.

Tangential velocity along the free stream is always equal to the nondimensional inlet velocity, 1. $\phi$, along the free stream must then always be equal to the arclength function defined previously, $s(r) . \partial \phi / \partial n$ along the streamline, or the flow normal to the surface, is by definition $0 . I_{f s}(\vec{r})$ can be written as follows:

$$
I_{f s}(\vec{r})=\int_{1}^{R_{p} / R_{j}}-s(r) \frac{\partial \mathcal{G}_{1}}{\partial n}(r, f(r)) d r
$$

where $f(r)$ is the $z$ value of the free surface as a function of $r$, found from eqn (27), and $\partial \mathcal{G}_{1} / \partial n$ is defined by:

$$
\frac{\partial \mathcal{G}_{1}}{\partial n}=\nabla \mathcal{G}_{1} \cdot \hat{n}
$$

where $\hat{n}$ is the unit normal vector to the free stream.

The final boundary, deflector plate itself, is situated at $z=0$ and spans a radius $0<r<R_{p} / R_{j}$. At $z=0, \partial \mathcal{G}_{1} / \partial n$ is always equal to 0 , meaning the potential along the boundary is irrelevant. Fluid can only pass through the plate at the location of the slot, meaning $\partial \phi / \partial n=0$ at all locations except the slot. By evaluating pressure through eqn (7) a velocity profile can be applied (see Steckler et al. [4]). $I_{p}(\vec{r})$ can be written as follows:

$$
I_{p}(\vec{r})=\int_{0}^{R_{p} / R_{j}} U_{s}(r) \mathcal{G}_{1}(r, 0) d r
$$


Where $U_{s}(r)$ is the velocity profile determined.

\subsection{Solution}

Applying the above boundary conditions produces a solution for the velocity potential, $\phi(\vec{r})$, but requires the guessing of the flow split, $\alpha$. The solution produced will accurately predict the results of the initial conditions. This can be corrected by iterating as follows. First, an approximate flow split based upon expected slot behavior is guessed, and the surface integral evaluated for all boundary conditions. When a solution for $\phi(\vec{r})$ is found, pressure can then be evaluated at the slot again through eqn (7). The newly evaluated pressure will produce a revised velocity profile, and by eqn (2), a new flow split predicted. The above steps are iterated until the flow split representative of the problem is converged upon.

\section{Conclusions}

A general model has been presented for determining the free surface flow field of a jet impinging on a perforated deflector plate. This canonical configuration has the essential features of the more complex deflector geometries found in real fire sprinklers. The model consists of posing the velocity potential as a boundary value problem and through the use of Green's functions, describing the entire flow field. The model was then specified to the axisymmetric case and formulation of the free stream equation explored.

Modeling the free surface flow provides important overall information describing the flow split between the radial flow leaving the deflector and the flow directed downward through the perforations. Furthermore, critical detailed information is provided by the model for predicting the drop characteristics comprising the initial sprinkler spray. The free streamline flow formulation is an attractive modeling alternative to Navier-Stokes based free surface CFD approaches where first-principle models are used along with level-set methods for treatment of the liquid-gas interface. The free streamline approach provides solution of the liquid streams through boundary evaluation without the stringent grid requirements that come from the need to resolve the liquid and gas flows at the interface and elsewhere (especially when the interface location is unknown).

Future work will consists of evaluating the effect of sheet characteristics in the axisymmetric configuration. Additional model development will include boundary value formulation to capture the periodic geometry (i.e. tines and slots) typical of real fire sprinklers

\section{References}

[1] D. Wu, D. Guillemin \& A. Marshall, A modeling basis for predicting the initial sprinkler spray. Fire Safety Journal, 42, pp. 283-294, 2007. 
[2] Lamb, H., Hydrodynamics. Dover Publications: New York, sixth edition edition, pp. 94-103, 1932.

[3] Batchelor, G., An Introduction to Fluid Dynamics. Cambridge University Press: New York, pp. 493-506, 1967.

[4] K. Steckler, H. Baum \& J. Quintiere, Fire induced flows through room openings-flow coefficients. Twentieth Symposium (International) on Combustion, The Combustion Institute: Pittsburg, pp. 1-26, 1985. 